\title{
Cross-Cultural Issues Concerning Sexuality, Fertility, and Childbirth
}

\author{
Jeannette E. South-Paul, MD
}

The discussion by Fisher and colleagues regarding naiveté and lack of exposure to issues of sexuality in South Asian Indians ${ }^{1}$ introduces a not uncommon problem that family physicians can encounter. The rapidly changing demographics of the US population and the surge of young immigrants - often professional-of childbearing age increase the chances that we will care for couples struggling with infertility.

The first step in evaluating infertility is always to interview the couple. The interviewing process for all patients proceeds more smoothly if it includes attention not only to the biopsychosocial model but also to the cultural model. Engel's biopsychosocial model emphasizes the importance of considering the whole patient and his or her experience of illness, rather than just a disease assessment. ${ }^{2}$ In contrast, the cultural model emphasizes that there are 2 persons contributing to any clinical encounter-the patient and the physician. Consequently, not only must the background and experiences of the patient be factored into the assessment but so must those of the physician.

American culture provides a wealth and breadth of exposure to its population that is unmatched in most countries around the world. Many aspects of that exposure, specifically the public manner in which private and intimate issues are discussed, establish an expectation of openness in all things personal, particularly with respect to the physicianpatient relationship. Even when there is respect for the clinical relationship and a desire to have a personal problem solved, the relatively naive patient might be unable to engage in an open discussion with a relative stranger.

Submitted 4 December 2002.

From the Department of Family Medicine, University of Pittsburgh, Pittsburgh. Address reprint requests to Jeannette E. South-Paul, MD, Department of Family Medicine, University of Pittsburgh, 3518 Fifth Ave, Pittsburgh, PA 15261.
The authors have accurately reported the paucity of literature regarding factors affecting the sexual expression of patients born and raised outside the United States. One study of student nurses and midwives in southern Africa, for instance, noted that $67 \%$ of married women had only ever had one sexual partner, although men were more sexually experienced. ${ }^{3}$ To pursue a discussion of issues of sexuality and fertility, the patient must have already catalogued and evaluated his or her symptoms to share them with the physician. Not only is the physician-patient discussion hampered, but also the discussion of such issues between the couple might be hampered by a lifetime of secrecy and cultural inhibition.

The situation described is undoubtedly not unique to South Asian Indians and relates to more than formal education. In fact, those immigrants with the family and financial resources to afford a formal education usually represent the more restricted segments of that society. As a result, formal education might not be the best proxy for worldliness. Rather, an interview that incorporates a social and cultural history to describe the personal world in which the patient was raised could be more revealing than questions regarding education and occupation, information that is often elicited before the physician even greets the patient. A study of sex knowledge and attitudes in Malaysian medical and nursing students showed that race, religion, age, perception of the importance of religion, and the extent to which religious attitudes influenced sexual attitudes were all significantly associated with sexual knowledge. ${ }^{4}$

One dilemma with attempting to describe all the unique social and cultural characteristics that might be encountered when seeing patients of other cultures is the risk of forming stereotypes rather than generalizations. In the case described by Drs. Fisher et al, a stereotype would be the expectation that all South Asian Indian women in the United 
States are sexually naïve and reluctant to reveal their inexperience with their physician. In contrast, a generalization would be the recognition that South Asian Indian women are often raised in a family environment much more restricted than is seen in the United States, so the physician must expand the scope of questions traditionally asked in the patient encounter. Furthermore, sexual inhibition can hamper or affect the willingness of patients to be seen by clinicians (including students) of the opposite sex. ${ }^{5}$

Many tools have been developed in recent years to assist clinicians in navigating the cross-cultural encounter. One of the earliest tools, the LEARN mnemonic, ${ }^{6}$ was developed by Berlin and Fowkes more than 15 years ago. Levin et $\mathrm{al}^{7}$ modified this earlier work to describe the ETHNIC model incorporating the concept of eliciting information about alternative healers. This model is useful in older patients as well. ${ }^{8}$ Stuart and Lieberman ${ }^{9}$ described the useful BATHE tool to elicit the emotional implications of the condition the patient is experiencing.

It would probably be even more helpful if we, as clinicians, used our own BATHE mnemonic to characterize how we feel when we face an unfamiliar patient who represents culture and custom foreign to our own experiences. Does the patient or the encounter leave us clueless, confused, concerned, or conflicted?

Clueless-the reason for the visit is not apparent despite careful questioning. Are there linguistic or literacy issues that might not have initially been apparent?

Confused-multiple problems seem present, but ability to prioritize is limited. Would the patient prioritize the seriousness of the problem in the same manner as the clinician?

Concerned-serious illness or disease is clear and requires appropriate planning to handle the problem in a culturally sensitive fashion.

Conflicted-the clinician feels uncomfortable, even angry, with aspects of the encounter that might relate to the patient's cultural values, what could be perceived as the patient's unrealistic expectations of the clinician or the system.
These items focus the clinician on personal reactions to the culturally foreign patient and assist in recognizing issues important to managing the patient's concerns, but which might not have been apparent during the initial history-taking process.

The case described by Fisher et al serves to remind us of our need to constantly practice cultural humility described by Tervalon and MurrayGarcia. ${ }^{10}$ Only when we are reminded consistently of our limitations when caring for those of varied cultures can we hope to attend appropriately to their needs and maintain a high-quality practice.

\section{References}

1. Fisher JA, Bowman M, Thomas T. Issues for South Asian Indian patients surrounding sexuality, fertility, and childbirth in the US health care system. J Am Board Fam Pract 2003;16:xxx-xx.

2. Engel GL. The need for a new medical model: a challenge for biomedicine. Science 1977;196:12936.

3. Verkuyl DA. Self-reported sexual and reproductive behavior of male and female student nurses and midwives in Bulawayo. Cent Afr J Med 2000;46:325-9.

4. Zulkifli SN, Low WY. Sexual health education for youths—a Malaysian experience. Asia Pac J Public Health 2000;12(Suppl):S58-66.

5. Rizk DE, Al-Shebah A, El-Zubeir MA, Thomas LB, Hassan MY, Ezimokhai M. Women's perceptions of and experiences with medical student involvement in outpatient obstetric and gynecologic care in the United Arab Emirates. Am J Obstet Gynecol 2002; 187:1091-100.

6. Berlin EA, Fowkes WC Jr. A teaching framework for cross-cultural health care. Application in family practice. West J Med 1983;139: 934-8.

7. Levin SR, Like RC, et al. 1997. ETHNIC: a framework for culturally competent clinical practice. $\mathrm{Pa}-$ tient Care 1997;9(Special Issue):188.

8. Kobylarz FA, Heath FM, Like RC. The ETHNIC(S) mnemonic: a clinical tool for ethnogeriatric education. J Am Geriatr Soc 2002;50:1582-9.

9. Stuart MR, Lieberman JA III. The fifteen minute hour: applied psychotherapy for the primary care physician. Westport, Conn: Praeger, 1993.

10. Tervalon M, Murray-Garcia J. Cultural humility versus cultural competence: a critical distinction in defining physician training outcomes in multicultural education. J Health Care Poor Underserved 1998;9:117-25. 